\title{
Effects of alkaline protease or restrictocin deficient mutants of Aspergillus fumigatus on human polymorphonuclear leukocytes
}

\author{
Y. Ikegami, R. Amitani, T. Murayama, R. Nawada, W.J. Lee, R. Kawanami, F. Kuze
}

Effects of alkaline protease or restrictocin deficient mutants of Aspergillus fumigatus on human polymorphonuclear leukocytes. Y. Ikegami, R. Amitani, T. Murayama, R. Nawada, W.J. Lee, R. Kawanami, F. Kuze. (OERS Journals Ltd 1998.

ABSTRACT: Several substances including proteases and restrictocin have been suggested as candidates for virulence determinants in invasive pulmonary aspergillosis. However, the roles of such substances are not well understood. This study compared the in vitro suppressive effects of Aspergillus fumigatus culture filtrates (ACFs), on the functions of human polymorphonuclear leukocytes (PMNLs), the principal cells in the host defence against aspergillus hyphae, from a clinically isolated wild-type and isogenic mutant strains which lack production of elastolytic alkaline protease (Alp) and/or restrictocin. ACFs were obtained by culturing conidia of each strain in Medium-199 at $37^{\circ} \mathrm{C}$ for 5 days.

ACFs of the wild-type significantly $(p<0.01)$ suppressed chemotaxis, superoxide anion $\left(\mathrm{O}_{2}^{-}\right)$release and PMNL-mediated hyphal damage, compared with the control (Medium-199). ACFs of the mutant strains that lack Alp or restrictocin significantly $(\mathrm{p}<0.01)$ suppressed chemotaxis and $\mathrm{O}_{2}^{-}$-release, but did not suppress hyphal damage, compared with the control. The wild-type significantly $(\mathrm{p}<0.01)$ suppressed chemotaxis of PMNLs compared with the mutant strains lacking Alp or restrictocin, whereas there were no significant differences in suppression of $\mathrm{O}_{2}^{-}$-release and hyphal damage by PMNLs. ACF of a mutant strain that lacks both Alp and restrictocin had much less activity, but significantly $(p<0.01)$ suppressed chemotaxis of PMNLs compared with the control.

In conclusion, alkaline protease and restrictocin may play roles in the suppressive effect of Aspergillus fumigatus culture filtrates on the functions of human polymorphonuclear leukocytes. Other antiphagocytic substances produced by Aspergillus fumigatus remain to be identified.

Eur Respir J 1998; 12: 607-611.
Dept of Infection and Inflammation, Chest Disease Research Institute, Kyoto University, Kyoto, Japan.

Correspondence: R. Amitani

Dept of Infection and Inflammation

Graduate School of Medicine

Kyoto University

Sakyo-ku

Kyoto 606-8397

Japan

Fax: 81757529017

Keywords: Alkaline protease

Aspergillus fumigatus

mutant strains

polymorphonuclear leukocyte

resrictocin

Received: July 101997

Accepted after revision April 71998

Presented at the ERS 1996 Annual Congress in Stockholm, Sweden, on September 91996
Aspergillus fumigatus is the most common pathogen in invasive pulmonary aspergillosis, but little is known of its pathogenic factors. Although several A. fumigatus-derived substances, including elastolytic alkaline protease (Alp), restrictocin and gliotoxin, have been suggested as candidates for virulence determinants of A. fumigatus [1-6], the role of each substance is poorly understood. Several research groups have constructed isogenic mutant strains which lack production of Alp or restrictocin from clinically isolated wild-types, by a gene disruption method, in order to elucidate the role of each substance in the pathogenesis of invasive aspergillosis. However, in vivo experiments using the mutant strains have not yet demonstrated the virulence determinants [7-12].

We previously reported that $A$. fumigatus culture filtrates (ACFs) suppressed functions of human alveolar macrophages and polymorphonuclear leukocytes (PMNLs), the principal cells in the host defence against aspergillus, and that multiple substances in the ACFs are likely to be responsible for the suppressive activities of the ACFs [13]. It was speculated that suppression of phagocytic functions would allow aspergillus to proliferate, presumably by escaping the local host defences.
In the present study the in vitro effects of ACFs on the functions of human PMNLs (chemotaxis towards formylmethionyl-leucyl-phenylalanine (FMLP), superoxide anion $\left(\mathrm{O}_{2}^{-}\right)$-release and hyphal damage by PMNLs) were compared between a clinically isolated $A$. fumigatus wild-type parent strain and its isogenic mutant strains which lack production of Alp and/or restrictocin, in order to evaluate the role of each substance in the suppression of PMNL functions.

\section{Materials and methods}

\section{Preparation of Aspergillus fumigatus culture filtrates}

A clinically isolated A. fumigatus strain and its isogenic mutant strains were a gift from D.W. Holden (Royal Postgraduate Medical School, London, UK). The A. fumigatus strains used in the present study were as follows: strain 237, a wild-type clinically isolated from a patient with invasive pulmonary aspergillosis; strain 342 (formerly called 237d), an Alp-deficient strain; strain 363, a restrictocin-deficient strain; strain 417 , a double-mutant strain lacking both Alp and restrictocin. The A. fumigatus 
mutant strains 342 and 363 were constructed from the wildtype strain 237 by single gene disruption [7-9]. Strain 417 was constructed by replacing the Alp gene in mutant strain 363 [10].

TANg et al. $[7,8]$ and SMith et al. $[9,10]$ confirmed disruption of the genes, as well as a lack of production of Alp and/or restrictocin, in the mutant strains. In contrast to the ACFs of the wild-type parent strain 237, the mutant strain 342 had no detectable proteolytic and elastolytic activities on azocollagen and elastin-Congo red, and sodium dodecyl sulphate-polyacrylamide gel electrophoresis (SDS-PAGE) of extracellular proteins from strain 342 showed the absence of a major $33 \mathrm{kDa}$ protein, which corresponds to Alp. Strain 363 lacks the specific extracellular ribonucleolytic activity associated with restrictocin, as measured by an in vitro rabbit reticulocyte lysate assay. Western blot analysis of strain 363, using anti-restrictocin monoclonal antibody, confirmed that it does not produce restrictocin. Strain 417 did not degrade elastin when grown on elastin medium, indicating the absence of Alp. Disruption of the restrictocin gene of strain 417 was confirmed by polymerase chain reaction analysis.

ACFs were obtained by the following procedures [13]. After growth on slants of potato dextrose agar (PDA) (Difco Laboratories, Detroit, MI, USA) for 5 days at $30^{\circ} \mathrm{C}$, conidia $\left(2 \times 10^{7}\right)$ from of each strain were cultured in flasks containing $400 \mathrm{~mL}$ of Medium-199 (Gibco BRL, Paisley, UK) cell culture fluid without shaking for 5 days at $37^{\circ} \mathrm{C}$. The fungal mass was removed by passing the medium through gauze and each culture filtrate was sterilized by passage through Millex-GV $0.22 \mu \mathrm{m}$ filters (Japan Millipore, Tokyo, Japan) and stored at $-80^{\circ} \mathrm{C}$. Before use in experiments, the $\mathrm{pH}$ of the ACFs was adjusted to 7.4 with $1 \mathrm{M} \mathrm{HCl}$. The sterility of the ACFs was tested by 3 days of culture on plates of blood agar number 2 (Oxoid, Basingstoke, UK) and PDA at $37^{\circ} \mathrm{C}$. During the 5 days of culture in Medium-199, growth rates of the mutant strains were indistinguishable from that of the wild-type parent strain. In addition, the protein concentrations of the ACFs, measured by Lowry's method, were 3.83, 4.07, 3.87 and 3.98 $\mu \mathrm{g} \cdot \mathrm{mL}^{-1}$ for strains $237,342,363$ and 417 , respectively.

\section{Cell preparations}

Fresh human PMNLs were prepared from heparinized venous blood obtained from 12 healthy adult volunteers by sedimentation in $2 \%$ dextran, followed by centrifugation on a Ficoll gradient and lysis of erythrocytes with hypotonic saline. The PMNLs were washed and resuspended in Medium-199 in polypropylene tubes (Falcon, Becton \& Dickinson Co., Lincoln, NJ, USA). Giemsa-stained cytospin preparations of the PMNL suspensions typically showed $>95 \%$ PMNLs. In each experiment, cell viability, assessed by the trypan-blue-exclusion test, was found to be $>90 \%$ after treatment with ACFs of each strain for $2 \mathrm{~h}$.

\section{Chemotaxis assay}

Chemotaxis of PMNLs under agarose was determined according to the method of NeLSON et al. [14]. In brief, six series of three wells, $3 \mathrm{~mm}$ in diameter and $3 \mathrm{~mm}$ apart, were cut in agarose plates. FMLP, $\left(10^{-7} \mathrm{M}\right.$; Sigma, St Louis, MO, USA) and Hank's balanced salt solution
(HBSS), $10 \mu \mathrm{L}$ of each, were placed in the outer and inner wells, respectively. The centre wells contained $1 \times 10^{6}$ PMNLs suspended in Medium-199 with ACFs (0, 3, 5, $10,20,30$ and $50 \%$ of each strain). The plates were incubated in a $5 \% \mathrm{CO}_{2}$ incubator for $2 \mathrm{~h}$ at $37^{\circ} \mathrm{C}$ and then fixed with methanol and stained with Giemsa. Migration was quantified by the linear distance that the cells had moved from the margin of the centre well towards the FMLP.

\section{Superoxide anion assay}

Production of $\mathrm{O}_{2}^{-}$was assayed by determining the superoxide dismutase (SOD)-inhibitable reduction of cytochrome $c$ [15]. In brief, $2.5 \times 10^{5} \mathrm{PMNLs}$ were preincubated in Medium-199 containing various concentrations of ACFs $(0,3,6,12.5,25$ and $50 \%)$ for $60 \mathrm{~min}$ at $37^{\circ} \mathrm{C}$ and then washed with phosphate-buffered saline (PBS). PMNLs were then incubated in a final volume of $500 \mu \mathrm{L}$ of HBSS containing cytochrome $c(120 \mu \mathrm{M})$ (Sigma), with or without $50 \mu \mathrm{g}$ SOD and phorbol myristate acetate (PMA, 100 $\left.\mathrm{ng} \cdot \mathrm{mL}^{-1}\right)$ (Sigma), in a shaking water bath for $30 \mathrm{~min}$ at $37^{\circ} \mathrm{C}$. The cells were finally removed by centrifugation. $\mathrm{O}_{2}^{-}$production in the supernatants was assessed as the difference in absorption between them and the control, at $550 \mathrm{~nm}$, measured using a spectrophotometer (Hitachi U3210 , Ibaragi, Japan), and the amount of $\mathrm{O}_{2}^{--}$-released in nmol $10^{6}$ PMNLs $^{-1}$ was calculated.

\section{Hyphal damage}

Hyphal damage caused by PMNLs was assessed by a colorimetric assay as described below. This method was reported by Meshulam et al. [16] as a quick and quantitative technique for the assessment of fungal cell damage.

Organism. Conidia of A. fumigatus strain $237\left(1.5 \times 10^{6}\right)$ were suspended in RPMI-1640 (Gibco BRL) containing $10 \mathrm{mM}$ N-2-hydroxyethylpiperazine-N-2-ethanesulphonic acid (HEPES) buffer, and then incubated in a polypropylene tube (Falcon, Becton \& Dickinson) for $16 \mathrm{~h}$ at $30^{\circ} \mathrm{C}$ and for an additional $2 \mathrm{~h}$ at $37^{\circ} \mathrm{C}$ to form hyphae.

Chemicals. 2,3-Bis-(2-methoxy-4-nitro-5-sulphonyl)-(2H)tetrazolium-5-carboxanilide (XTT) (Sigma) was freshly prepared daily in Dulbecco's PBS (Gibco BRL) at 0.5 $\mathrm{mg} \cdot \mathrm{mL}^{-1}$ with heating at $60^{\circ} \mathrm{C}$ for $30 \mathrm{~min}$, and then passed through a $0.22 \mu \mathrm{m}$ filter prior to use. Coenzyme Q (2,3dimethoxy-5-methyl-1,4-benzoquinone) (Sigma) was prepared as a $5 \mathrm{mg} \cdot \mathrm{mL}^{-1}$ stock solution in PBS, and then filtered and stored in aliquots at $-70^{\circ} \mathrm{C}$. Working solutions prepared for each experiment contained $0.5 \mathrm{mg} \cdot \mathrm{mL}^{-1} \mathrm{XTT}$ and $40 \mu \mathrm{g} \cdot \mathrm{mL}^{-1}$ coenzyme $\mathrm{Q}$.

Assessment of hyphal damage. Human PMNLs were preincubated in Medium-199 with or without 30\% ACF from each strain for $1 \mathrm{~h}$ at $37^{\circ} \mathrm{C}$. Hyphae in quadruplicate polypropylene tubes were sedimented by centrifugation and resuspended in $600 \mu \mathrm{L}$ PBS supplemented with $1 \mathrm{~g} \cdot \mathrm{L}^{-1} \mathrm{glu}-$ cose, $100 \mathrm{mg} \cdot \mathrm{mL}^{-1} \mathrm{Ca}^{2+}$ and $100 \mathrm{mg} \cdot \mathrm{mL}^{-1} \mathrm{Mg}^{2+}$. PMNLs $\left(6 \times 10^{6}\right)$ in a final volume of $1 \mathrm{~mL}$ of supplemented PBS with $10 \%$ heat-inactivated human blood group $\mathrm{AB}$ serum were added to the tubes. Two controls were incubated in each experiment: one set of the tubes containing fungi and serum and the other containing PMNLs and serum. The 
tubes were incubated for $1 \mathrm{~h}$ at $37^{\circ} \mathrm{C}$ in a $5 \% \mathrm{CO}_{2}$ incubator. PMNLs were lysed by adding cold distilled water, followed by centrifugation at $4^{\circ} \mathrm{C}$. Supernatants were aspirated and the pellets were washed with $1 \mathrm{~mL}$ distilled water and then resuspended in $0.4 \mathrm{~mL}$ working solution containing XTT and coenzyme Q, and incubated for $1 \mathrm{~h}$ at $37^{\circ} \mathrm{C}$. An aliquot $(100 \mu \mathrm{L})$ from each supernatant was then transferred to a well of a microtitre plate (Falcon, Becton \& Dickinson). Absorption at $450 \mathrm{~nm}$ was determined using a 96-well enzyme-linked immunosorbent assay (ELISA) plate reader (Flow Laboratories, Dainippon Pharmaceutical Co., Ltd., Suita, Japan). The percentage of hyphal damage was defined by the following equation:

$$
\left[1-\frac{A_{450} \text { of fungi incubated with PMNLs - }}{A_{450} \text { of fungi incubated without PMNLs }}\right] \times 100(\%)
$$

\section{Statistical analysis}

All results are expressed as the mean \pm SEM. The data were analysed using the statistical software package Statview 4.11 (Abacus Concepts, Berkeley, CA, USA). Comparison was performed with the Kruskal-Wallis test and then multiple comparisons were performed by Bonferroni's method. A p-value $<0.05$ was considered to be significant.

\section{Results}

Effects of Aspergillus fumigatus culture filtrates on chemotaxis of human polymorphonuclear leukocytes

The effects of ACF on the chemotaxis of human PMNLs are shown in figure 1 . ACF of strain 237 significantly $(\mathrm{p}<0.01)$ suppressed the migration of PMNLs tow-

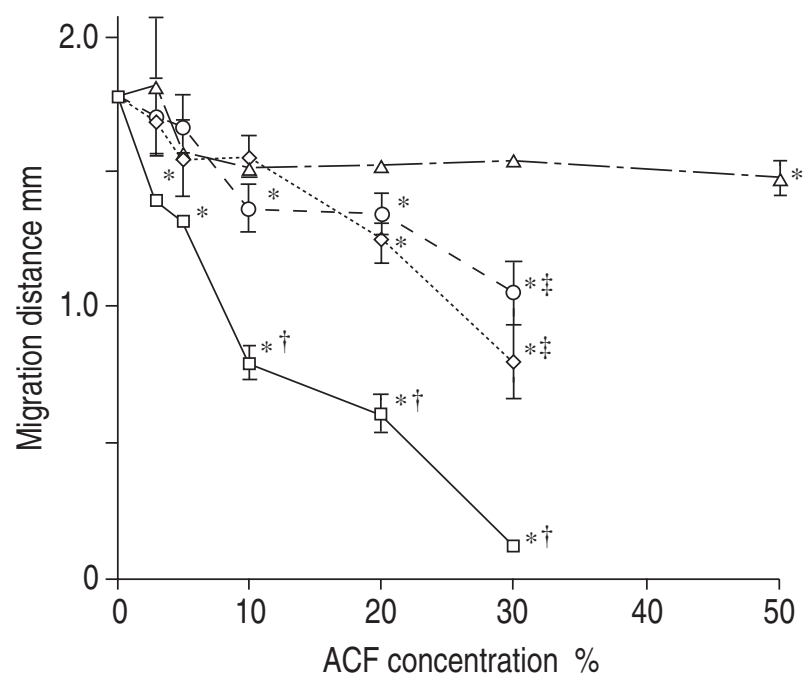

Fig. 1. - Effect of Aspergillus fumigatus culture filtrates (ACFs) on chemotaxis of human polymorphonuclear leukocytes (PMNLs). Chemotactic activity was expressed as the linear distance $(\mathrm{mm})$ which the cells migrated towards formyl-methionyl-leucyl-phenylalanine $\left(10^{-7} \mathrm{M}\right)$. The migration distances of PMNLs treated with various concentrations of ACFs from wild-type ( $\square$, strain 237) and mutant strains $(\diamond$, strain $342 ; 0,363 ; \Delta, 417$ ) were plotted. The experiments were repeated four times, each performed in quadruplicate, and data are presented as the mean \pm SEM. $*: p<0.01$, compared with control (Medium-199); ${ }^{\dagger}: \mathrm{p}<0.01$, compared with ACFs of strains 342,363 and 417; $\mathrm{p}<0.01$, compared with ACFs of strain 417. ards FMLP in a concentration-dependent manner between 3 and $30 \%$ compared to control (medium alone). At $30 \%$ strain 237 ACF, PMNLs were almost immotile. Compared with control, ACFs of strains 342 and 363 also significantly $(\mathrm{p}<0.01)$ suppressed migration of PMNLs at concentrations of $20-30 \%$ and $10-30 \%$, respectively. The suppressive effect of ACF of strain 417 was significant $(p<0.01)$ at a concentration of $50 \%$, but not at $3-30 \%$, compared with control. ACF of strain 237 significantly $(\mathrm{p}<0.01)$ suppressed migration of PMNLs compared with ACFs of strains 342,363 and 417 at concentrations of 10-30\%. ACFs of strains 342 and 363 also significantly $(\mathrm{p}<0.01)$ suppressed migration of PMNLs compared with strain 417 ACF at a concentration of $30 \%$. There were no significant differences in the suppressive effect between the ACFs of strains 342 and 363.

Effects of Aspergillus fumigatus culture filtrates on superoxide anion release from human polymorphonuclear leukocytes

ACFs of strain 237 significantly $(\mathrm{p}<0.01)$ suppressed $\mathrm{O}_{2}-$-release from PMNLs in response to PMA, compared with both control (medium alone) and ACFs of strain 417 at concentrations of 25-50\% (fig. 2). ACFs of strains 342 and 363 significantly $(\mathrm{p}<0.01)$ suppressed $\mathrm{O}_{2}-$-release at a concentration of $50 \%$ compared with both ACFs of strain 417 and control. ACFs of strain 417 did not show any significant changes. There were no significant differences in the suppressive effect on $\mathrm{O}_{2}^{-}$-release between ACFs of strains 342 and 363.

Effects of Aspergillus fumigatus culture filtrates on hyphal damage by human polymorphonuclear leukocytes

The percentage of hyphal damage by PMNLs pretreated with medium alone (control) was $77.2 \pm 1.6 \%$ (fig. 3).

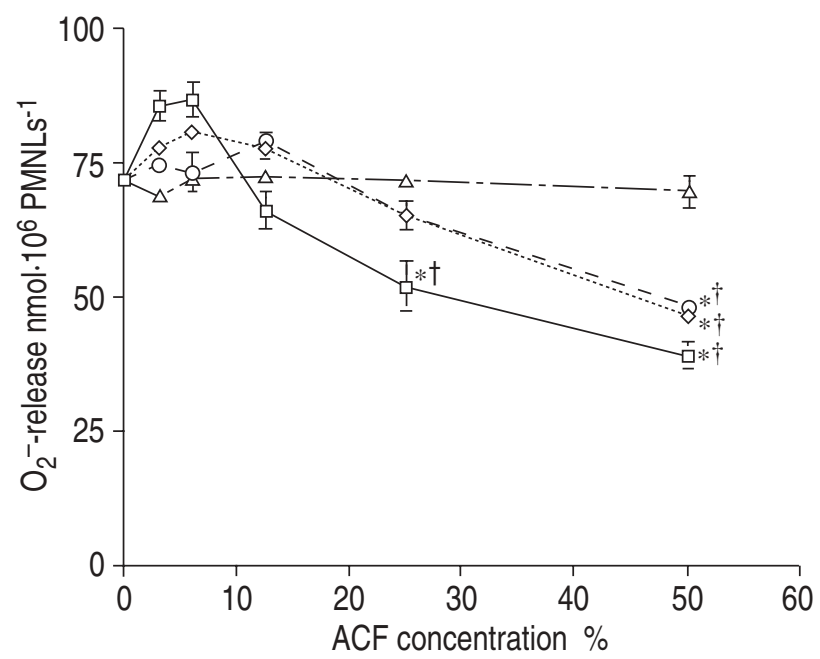

Fig. 2. - Effect of Aspergillus fumigatus culture filtrates (ACFs) on superoxide anion $\left(\mathrm{O}_{2}^{-}\right)$-release from human polymorphonuclear leukocytes (PMNLs) stimulated with phorbol myristate acetate $\left(100 \mathrm{ng} \cdot \mathrm{mL}^{-1}\right)$. $\mathrm{O}_{2}^{-}$-release (expressed as nmol $10^{6} \mathrm{PMNLs}^{-1}, 30 \mathrm{~min}$ ) from human PMNLs treated with various concentrations of ACFs of strains 237 ( $\square)$, $342(\diamond), 363(\circ)$ and $417(\Delta)$ were plotted. The experiments were repeated four times, each performed in quadruplicate, and data are expressed as the mean \pm SEM. $*: p<0.01$, compared with control (Medium-

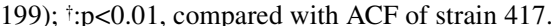




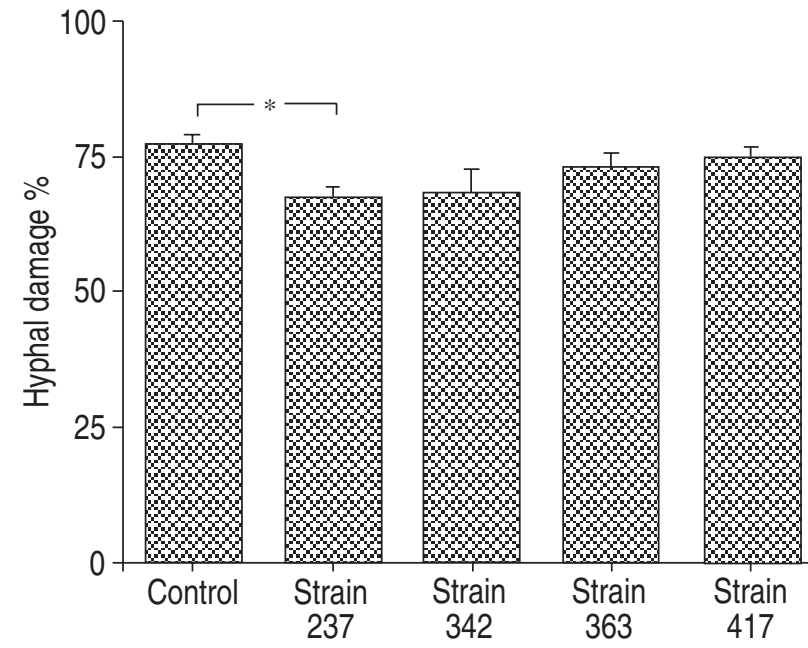

Fig. 3. - Effect of $30 \%$ Aspergillus fumigatus culture filtrates on polymorphonuclear leukocyte-mediated damage to A. fumigatus hyphae. The percentage of hyphal damage was examined by the 2,3-bis-(2-methoxy-4-nitro-5-sulphonyl)-(2H)-tetrazolium-5-carboxanilide (XTT) colorimetric assay. The experiments were repeated eight times, each performed in quadruplicate, and data are presented as the mean \pm SEM. $* \cdot p<0.01$, compared with control (Medium-199).

Pretreatment with $30 \%$ ACF of strain 237 significantly $(\mathrm{p}<0.01)$ reduced hyphal damage to $67.2 \pm 12.4 \%$, compared with control. Pretreatment with $30 \%$ ACF of mutant strains 342, 363 and 417 did not significantly reduce the percentage of hyphal damage by PMNLs $(68.1 \pm 4.0 \%$ (strain 342), $72.3 \pm 2.7 \%$ (strain 363) and $74.1 \pm 1.8 \%$ (strain 417)), compared with control. However, there were no significant differences between strains 237, 342, 363 and 417.

\section{Discussion}

The in vitro suppressive effects of ACFs on the functions of PMNLs were compared in a clinically isolated wild-type and its isogenic mutant strains which lack production of Alp and/or restrictocin. ACFs of the wild-type significantly suppressed chemotaxis and $\mathrm{O}_{2}{ }^{-}$-release by PMNLs and PMNL-mediated hyphal damage compared with control (Medium-199). ACFs of the mutant strains which lack production of Alp or restrictocin also significantly suppressed chemotaxis and $\mathrm{O}_{2}^{-}$-release by PMNLs, but did not suppress hyphal damage. The ACF of the wildtype had the highest suppressive activity of all strains examined in this study and significantly suppressed chemotaxis of PMNLs compared with the isogenic mutant strains lacking Alp or restrictocin. ACF of a mutant strain which lacks both Alp and restrictocin had much less activity, but significantly suppressed chemotaxis of PMNLs compared with control.

Several reports have suggested a pathogenic role for elastolytic Alp and restrictocin [1-4]. КотHARY et al. [2] reported that mice pretreated with cortisone acetate had a significantly higher mortality rate and greater extent of lesions in lung tissues when they inhaled conidia of elastolytic protease-producing strains compared with nonproducing strains. Restrictocin, an $18 \mathrm{kDa}$ protein belonging to the mitogillin family, is an A. fumigatus-derived cytotoxin with ribonucleolytic activity and has also been suggested to be a pathogenic factor in human invasive aspergillosis [4]. With the aim of evaluating the role and significance of Alp and restrictocin as virulence determinants in the pathogenesis of invasive pulmonary aspergillosis, several in vivo studies have been performed using A. fumigatus mutant strains lacking production of either of these substances. However, in spite of the in vivo studies using the mutant strains, the role and the significance of each substance are still controversial [7-12]. The animal models used in these studies were acute invasive aspergillosis models that had been severely immunosuppressed. This may be one reason for the lack of differences.

It has been considered that human host defences against A. fumigatus consist of phagocytosis and killing of conidia by alveolar macrophages to prevent germination and PMNL-mediated hyphal damage induced by the release of reactive oxygen intermediates and cationic peptides [1720]. In particular, PMNLs are considered to play a vital role in the defence against $A$. fumigatus because, in human tissues, A. fumigatus grows almost exclusively in the mycelial form. It has also been considered that the pathogenesis of invasive aspergillosis is closely related to impairment of the host defence, especially PMNL-function. It was speculated that $A$. fumigatus produces substances that suppress the functions of PMNLs and that the suppressive effects make it easier for A. fumigatus to proliferate and invade the surrounding tissues. From this point of view, the effects of ACFs on the functions of PMNLs were investigated.

ROBERTSON et al. [21-23] have already reported that $A$. fumigatus spore diffusates obtained from $3 \mathrm{~h}$ culture supernatants suppressed the migration of human PMNLs and the spontaneous $\mathrm{O}_{2}^{-}$-release from mouse peritoneal exudate cells and bronchoalveolar lavage cells in Corynebacterium Parvarum treated rats. ACF contains multiple substances that suppress migration of human PMNLs to-

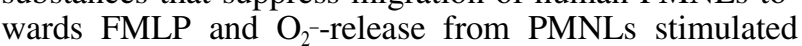
with PMA. The antiphagocytic substances remain to be identified [13].

The results of the present study indicate that Alp and restrictocin play roles in the suppression of human PMNL functions and that other substances also have suppressive effects. Although it is very important to evaluate the suppressive effects of purified Alp and restrictocin on the functions of PMNLs, the present study using a wild strain and isogenic mutant strains which lack production of a single substance should help to evaluate the role and significance of each substance involved in the suppressive activity of ACFs.

Two points are worth considering in regard to the use of ACFs in the present study. The first is whether the growth rates of the wild and the mutant strains cultured in Medium-199 were the same. The appearance of the growth in the medium suggested that very similar growth rates were obtained throughout the 5 days of culture. In addition, similar values were shown for protein concentrations in the ACFs of the strains, which supports almost identical growth rates. The other point worth considering is whether there was only a deficiency in the production of Alp or restrictocin in each strain generated by gene disruption. TANG, SMITH, Holden and coworkers [7-10] demonstra-ted the deficiency of Alp and/or restrictocin in ACFs of the mutants, but no differences in SDS-PAGE analysis, other than the deficient substance, between the wild and the 
mutant strains. However, whether or not gene disruption alters the properties of $A$. fumigatus and induces an ability to produce substances in human tissues that can compensate for the deficient substance still remains to be elucidated.

In conclusion elastolytic alkaline protease and restrictocin produced by Aspergillus fumigatus play roles in suppressing the functions of human polymorphonuclear leukocytes, and other antiphagocytic substances produced by Aspergillus fumigatus remain to be identified.

\begin{abstract}
Acknowledgements: The authors thank D.W. Holden, Dept of Infectious Diseases and Bacteriology, Royal Postgraduate Medical School, London, for providing the mutant strains of A. fumigatus, R. Diamond, Section of Infectious Diseases, Boston University Medical Center, Boston, for his helpful advice on XTT colorimetric assay and S. Oguma for his generous advice on statistical analysis. The authors are grateful to T. Homma, T. Ueda and K. Kataoka for their skilled assistance.
\end{abstract}

\section{References}

1. Miyaji M, Nishimura K. Relationship between proteolytic activity of Aspergillus fumigatus and the fungus' invasiveness of mouse brain. Mycopathologia 1977; 62: 161-166.

2. Kothary MH, Chase T Jr, MacMillian JD. Correlation of elastase production by some strains of Aspergillus fumigatus with ability to cause pulmonary invasive aspergillosis in mice. Infect Immun 1984; 43: 320-325.

3. Rhodes JC, Bode RB, McCuran-Kirsh CM. Elastase production in clinical isolates of Aspergillus. Diagn Microbial Infect Dis 1988; 10: 165-170.

4. Lamy B, Moutaouakil M, Latge J-P, Davies J. Secretion of a potential virulence factor, a fungal ribonucleotoxin, during human aspergillosis infections. Molec Microbiol 1991; 5: 1811-1815.

5. Mullbacher A, Waring P, Eichner RD. Identification of an agent in cultures of Aspergillus fumigatus displaying antiphagocytic and immunomodulating activity in vitro. $J$ Gen Microbiol 1985; 131: 1251-1258.

6. Amitani R, Taylor G, Elezis E-N, et al. Purification and characterization of factors produced by Aspergillus fumigatus which affect human ciliated respiratory epthelium. Infect Immun 1995; 63: 3266-3271.

7. Tang CM, Cohen J, Holden DW. An Aspergillus fumigatus alkaline protease mutant constructed by gene disruption is deficient in extracellular elastase activity. Molec Microbiol 1992; 6: 1663-1671.

8. Tang CM, Cohen J, Krausz T, Noorden SV, Holden DW. The alkaline protease of Aspergillus fumigatus is not a virulence determinant in two murine models of invasive pulmonary aspergillosis. Infect Immun 1993; 61: 16501656.

9. Smith JM, Davies JE, Holden DW. Construction and pathogenicity of Aspergillus fumigatus mutants that do not produce the ribotoxin restrictocin. Molec Microbiol 1993; 9: 1071-1077.

10. Smith JM, Tang CM, Noorden SV, Holden DW. Virulence of Aspergillus fumigatus double mutants lacking restrictocin and an alkaline protease in low-dose model of invasive pulmonary aspergillosis. Infect Immun 1994; 62: 5247-5254.

11. Monod M, Paris S, Sarfati J, Jaton-Ogay K, Ave P, Latge JP. Virulence of alkaline protease-deficient mutants of Aspergillus fumigatus. FEMS Microbiol Lett 1993; 106: $39-46$.

12. Kolattukudy PE, Lee JD, Rogers LM, et al. Evidence for possible involvement of an elastolytic serine protease in aspergillosis. Infect Immun 1993; 61: 2357-2368.

13. Murayama T, Amitani R, Ikegami Y, Nawada R, Lee WJ, Kuze F. Suppressive effects of Aspergillus fumigatus culture filtrates on human alveolar macrophages and polymorphonuclear leukocytes. Eur Respir J 1996; 9: 293-300.

14. Nelson RD, Quie PG, Simmons RL. Chemotaxis under agarose: a new and simple method for measuring chemotaxis and spontaneous migration of human polymorphonuclear leukocytes and monocytes. J Immunol 1975; 115: 1650-1656.

15. Johnston RB Jr, Godzik CA, Cohn ZA. Increased superoxide anion production by immunologically activated and chemically elicited macrophages. J Exp Med 1978; 148: $115-127$.

16. Meshulam T, Levitz SM, Christin L, Diamond RD. A sim-plified new assay for assessment of fungal cell damage with the tetrazolium dye, (2,3)-bis-(2-methoxy-4-nitro-5-

sulfonyl)-(2H)-tetrazolium-5-carboxanilide (XTT). J Infect Dis 1995; 172: 1153-1156.

17. Schaffner A, Douglas H, Braude A. Selective protection against conidia by mononuclear and against mycelia by polymorphonuclear phagocytes in resistance to Aspergillus. J Clin Invest 1982; 69: 617-631.

18. Schaffner A. Macrophage-Aspergillus interactions. Iтmиnol Ser 1994; 60: 545-552.

19. Diamond RD, Clark RA. Damage to Aspergillus fumigatus and Rhizopus oryzae hyphae by oxidative and nonoxidative microbiocidal products of human neutrophils in vitro. Infect Immun 1982; 38: 487-495.

20. Levitz SM, Selsted ME, Ganz T, Lehrner RI, Diamond RD. In vitro killing of spores and hyphae of Aspergillus fumigatus and Rhizopus oryzae by rabbit neutrophil cationic peptides and bronchoalveolar macrophages. $J$ Infect Dis 1986; 154: 483-489.

21. Robertson MD, Seaton A, Milne L Jr, Raeburn JA. Resistance of spores of Aspergillus fumigatus to ingestion by phagocytic cells. Thorax 1987; 42: 466-472.

22. Robertson MD, Seaton A, Milne L Jr, Raeburn JA. Suppression of host defence by Aspergillus fumigatus. Tho$\operatorname{rax} 1987$; 42: 19-25.

23. Robertson MD, Seaton A, Raeburn JA, Milne L Jr. Inhibition of phagocyte migration and spreading by spore diffusates of Aspergillus fumigatus. J Med Ver Myco 1987; 25: 389-396. 